V.Z. S.Z. OH.Z.J.Z.

1. Sdp.12 123 bis $128^{\circ}, 6,0 \mathrm{~g}$, farbl. fl. - - - -

2. 128 bis $130^{\circ}, 16,4 \mathrm{~g}$, farbl. fl. $332,10,2202,60,6$

3. $\quad 130^{\circ}, \quad 16,0 \mathrm{~g}$, farbl. fl. $341,10,2229,71,0$

4. Rückstand $2,0 \mathrm{~g}$, gelb, $\mathrm{fl}$.

Für Monoacetylester berechnete V.Z. $=\mathrm{OH}-\mathrm{Z} .=326,2$.

\section{Hexahydrosaligenin-dibenzoat}

70,5 g Hexahydrosaligenin (Sdp.12 136 bis $139^{\circ}, \mathrm{OH}-$ Z. 740,4, verunreinigt durch Hexahydrophthalanol) werden mit $152 \mathrm{~g}$ Benzoylchlorid und $350 \mathrm{~g}$ Pyridin, wie vorstehend beschrieben, umgesetzt und aufgearbeitet. Neben niedriger siedenden Anteilen erhält man folgende Hauptfraktion:
Sdp. ${ }_{0,3} 210$ bis $215^{\circ}, 95 \mathrm{~g}$, farbl. zähfl., V.Z. 323,5 (Theorie 332,0), S.Z. 3,0, OH-Z. 0, J.Z. 0,5.

\section{Hexahydrosaligenin-dihexahydrobenzoat}

$130 \mathrm{~g}$ Hexahydrosaligenin (Sdp.12 133 bis $135^{\circ}, \mathrm{OH}-\mathrm{Z}$. 547,4 , verunreinigt durch Hexahydrophthalanol), $295 \mathrm{~g}$ Hexahydrobenzoylchlorid und $645 \mathrm{~g}$ Pyridin werden, wie beim Acetylchloridansatz beschrieben, umgesetzt und aufgearbeitet. Nach wiederholter Destillation erhält man neben niedriger siedenden Produkten $74 \mathrm{~g}$ eines gelben, dickflüssigen Öles vom $\operatorname{Sdp}_{0,1} 186^{\circ}$, V.Z. 284,6 (Theorie 320,6), S.Z. 0,4, OH-Z. 4,9, J.Z. 0,3.

An dieser Stelle danken wir Frl. Gertrud Merkel für die Ausführung der chemischen Kennzahlanalysen.

\title{
Über die Umsetzung von Formaldehyd mit Äthylen
}

\author{
Von Sigurd Olsen \\ Aus dem Allg. Chem. Universitätslaboratorium Göttingen \\ (Z. Naturforschg. 1, 676-682 [1946]; eingegangen am 4. Juni 1946)
}

\begin{abstract}
Das Äthylen reagiert mit Formaldehyd in Gegenwart von Eisessig und Schwefelsäure unter Bildung von Trimethylenglykol-diacetat und Pentaglycerin-triacetat. Das Trimethylenglykol-diacetat läßt sich durch Destillation mit Schwefelsäure oder durch einen Crackprozeß in Allylacetat überführen. Dieses liefert beim Behandeln mit Formaldehyd höhersiedende Ester, die wahrscheinlich als perhydrierte Furanderivate anzusprechen sind.
\end{abstract}

\begin{abstract}
$A$ us unseren Arbeiten über die Umsetzung von Formaldehyd mit Cyclohexen ${ }^{1,2}$ hatten sich Fragen ergeben, die uns dazu führten, rein aliphatische Olefine, und zwar zuerst das Äthylen, auf Analogien zu prüfen. Für diesen $\mathrm{Zweck}$ wäre freilich ein höhersiedendes Olefin wegen der einfacheren präparativen Handhabung geeigneter gewesen, jedoch verfolgten wir gleichzeitig das weiter gesteckte Ziel, von dem bei der Reaktion zu erwartenden Trimethylenglykol aus zum Glycerin zu gelangen.

Wenn das Äthylen mit Formaldehyd in Eisessig unter dem katalytischen Einfluß von Schwefelsäure dem Cyclohexen analog reagiert, könnten folgende Reaktionsprodukte entstehen: 2.5-Dihydro-furan, Äthylacetat, Allylacetat, 1.3-Dioxan, 3-Acetoxy-tetrahydrofuran und Trimethylenglykol-diacetat.

1 O ls en u. Padberg, Z. Naturforschg. 1, 448 [1946].

2 Olsen, Z. Naturforschg. 1, 671 [1946].
\end{abstract}

\section{De r A t oklavenversuch}

Die Reaktion, die zur Erreichung einer mög lichst hohen Äthylenkonzentration unter Druck ausgeführt wurde', ergab unter den niedriger siedenden Anteilen eine Fraktion vom Sdp.752 56 bis $58^{\circ}$, die noch nicht sicher identifiziert worden ist. Weitere niedrigsiedende Produkte bis zum Siedepunkt des 1.3-Dioxans (Sdp. $106^{\circ}$ ), insbesondere Allylacetat, wurden nicht gefunden. Es ist möglich, daß das 1.3-Dioxan beim Waschprozeß verlorengegangen ist. Aus den höhersiedenden Fraktionen wurden isoliert: 1. Trimethylenglykoldiacetat (II), das in das freie Trimethylenglykol übergeführt wurde; dieses wurde als Bisphenylurethan identifiziert. 2. Pentaglycerin-triacetat (III), aus dem freies Pentaglycerin dargestellt wurde. Als Leitfaden bei der Entscheidung über

3 Leider stand uns komprimiertes Äthylen nur für wenige Ansätze zur Verfügung, weshalb wir uns bezüglich der Verwendung des aus der Synthese gewonnenen Materials größte Beschränkungen auferlegen mußten. 
die Konstitution des letzteren diente folgende Úberlegung: Einerseits gibt es mehrere Beispiele für den Übergang des Trimethylenglykols in Propionaldehyd ${ }^{4}$. Andererseits haben $\mathrm{Hos}$ a e u s ${ }^{5}$ sowie Koch und Zerner ${ }^{6}$ diesen durch alkalische Kondensation mit Formaldehyd nach der Tolle n s schen Reaktion in 2-Methyl-2-methylolpropandiol-(1.3) oder Pentaglycerin übergeführt. Hiermit ist jedoch noch nicht gesagt, daß die Reaktion tatsächlich diesen Verlauf nimmt. Unsere Substanz stimmte mit derjenigen von $\mathrm{H}$ os a e u s sowohl im Schmelzpunkt als auch in der Fähigkeit zur Sublimation und zur Bildung eines Triacetates überein, so daß an der Identität beider Substanzen nicht zu zweifeln war. Das wesentliche Ergebnis der Drucksynthese sei daher vorläufig durch die Formelfolge I $\rightarrow$ II + III wiedergegeben.

Da sich aus dem Druckversuch keine weiteren Einzelheiten über den Reaktionsverlauf entnehmen ließen, sollten nachstehende Versuche weiteren Aufschluß geben.

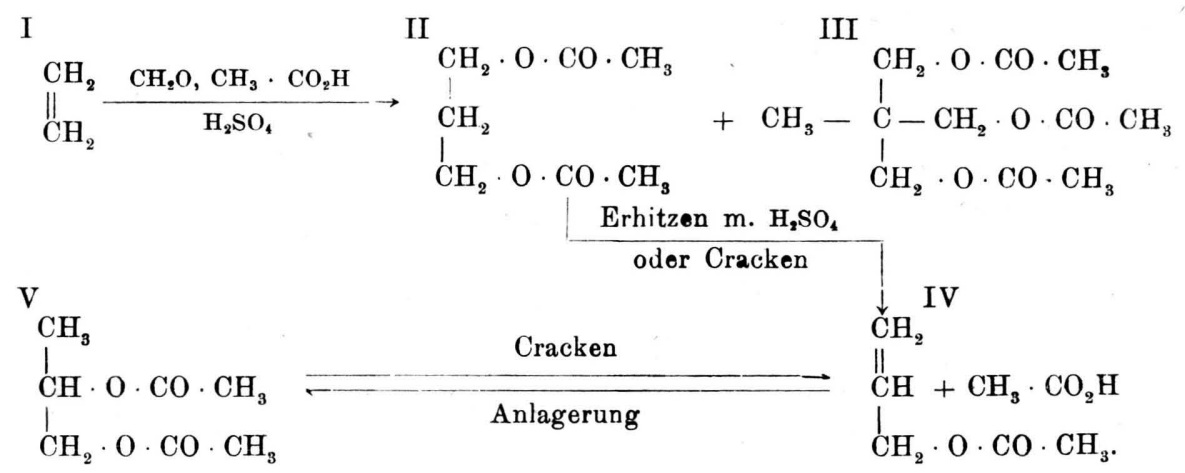

\section{Gleichgewichtsuntersuchung}

Wir haben uns zunächst gefragt, ob das zwischen Tetrahydrobenzylacetat und Hexahydrosaligenin-diacetat beobachtete Gleichgewicht ${ }^{1}$ auch für das Paar Allylacetat-Trimethylenglykol-diacetat (II $\rightleftharpoons$ IV) Gültigkeit besitzt. Aus den Arbeiten von Urushibara und Takebayashi ${ }^{7}$ sowie $\mathrm{Kh}$ arasch ${ }^{8}$ geht hervor, daß die Anlagerung von Bromwasserstoff an Allylbromid durch Innehaltung bestimmter Versuchsbedingungen entweder bevorzugt zum 1.2- oder zum 1.3-Dibrompropan führt. Mit ähnlichen Verhältnissen wäre grundsätzlich auch bei der Anlagerung von Essigsäure an Allylacetat zu rechnen. Wir haben durch Erhitzen von Trimethylenglykol-diacetat mit einer geringen Menge Schwefelsäure Allylacetat erhalten. Umgekehrt konnten wir bei dem Versuch, Essigsäure an Allylacetat durch Erhitzen im Rohr

1 Nef, Liebigs Ann. Chem. 335, 206; Chem. Zbl. 1904 II, 1139; R ix, Mh. Chem. 25, 273; Chem. Zbl. 1904 I, 1401; N e u be r g u. R e w ald, Biochem. Z. 71, 163; Chem. Zbl. 1915 II, 879.

$5 \mathrm{H}$ o s a e us, Liebigs Ann. Chem. 276, 76; Chem. Zbl. 1893 II, 528.

- K o c h u. Z e r n e r, Mh. Chem. 22, 443; Chem. Zbl. 1901 II, 525. anzulagern, in Übereinstimmung mit B é h a 1 und Desgrez ${ }^{\bullet}$ jedoch nur Propylenglykol-diacetat (V) isolieren. Auch Zusätze wie Schwefelsäure oder Ferrosulfat vermochten an diesem Reaktionsverlauf nichts zu ändern.

\section{Umsetzung von Allylacetat mit Formaldehyd}

In Analogie zu der Umsetzung von Tetrahydrobenzylacetat mit Formaldehyd in Eisessig ${ }^{1}$, müßte die entsprechende Umsetzung des Allylacetats bzw. Allylalkohols zum 2.5-Dihydro-furan, 3-Acetoxytetrahydro-furan (VI) und Trimethylenglykoldiacetat (II) führen. Die Reaktion lieferte zwei einheitliche Fraktionen $\operatorname{Sdp}_{10} 63,2$ bis $63,5^{\circ}$ und $\operatorname{Sdp}_{\cdot_{10.5}} 146$ bis $146,5^{\circ}$. Bei der niedriger siedenden Fraktion von der Formel $\mathrm{C}_{6} \mathrm{H}_{10} \mathrm{O}_{3}$ handelt es sich vermutlich um 3-Acetoxy-tetrahydro-

7 Urushibara u. Take bay a s h i, Bull. chem. Soc. Japan 11, 692, 754, 798 [1936]; 12, 51, 138, 173 [1937] ; 13, 400 [1938] ; Chem. Zbl. 1938 I, 3453; 1939 I, 89.

$8 \mathrm{~K} \mathrm{~h}$ a r a s c h, vergl. A. PP. 2058465 und 2058466 v. 16. Dez. 1933; Chem. Zbl. 1937 I, 4293.

9 B é h a l u. D e s g r e z, C. r. hebd. Séances Acad. Sci. 114, 676; Ber. dtsch. chem. Ges. 25 (Ref.), 463 [1892]. 
furan (VI). Der höhersiedenden Fraktion $\mathrm{C}_{9} \mathrm{H}_{14} \mathrm{O}_{5}$ dürfte vielleicht die Konstitution des 3-Acetoxy4-acetoxymethyl-tetrahydrofurans (VII) zukommen. Die Bildung beider Verbindungen läßt sich durch folgendes Reaktionsschema veranschaulichen:

A) Zuerst erfolgt Veresterung des Allylalkohols zum Allylacetat.

B) Das Allylacetat setzt sich mit Formaldehyd acetates um:

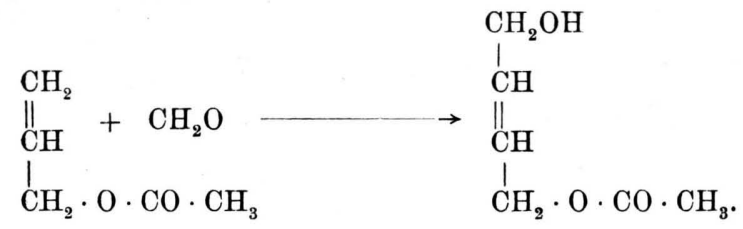

C) Das Buten-(2)-diol-(1.4) -monoacetat kann verschiedenartig reagieren:

1. kann es an seine olefinische Doppelbindung Essigsäure addieren: unter Bildung des Buten-(2)-diol-(1.4)-mono-

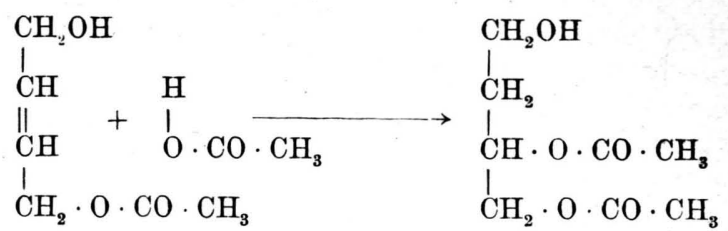

Das so entstandene Butantriol-(1.3.4)-diacetat geht unter den vorliegenden Bedingungen wegen der Möglichkeit zur Fünfringbildung in das 3-Acetoxy-tetrahydrofuran (VI) über :<smiles>COC(=O)OC(C)CC(C)OC(C)=O</smiles>

2. kann sich das Buten-(2)-diol-(1.4)-monoacetat nach dem Prinzip der Prinsschen Reaktion ${ }^{\mathbf{1 0}}$ gleichzeitig mit Formaldehyd und Essigsäure unter Bildung des 2-Oxymethylbutantriol-(1.3.4)-diacetates umsetzen, das. dann unter Ringschluß und Veresterung in das 3-Acetoxy-4-acetoxymethyl-tetrahydrofuran (VII) übergeht:

$$
\begin{aligned}
& \mathrm{HC}=\mathrm{CH}+\mathrm{CH}_{2} \mathrm{O}+\mathrm{CH}_{3} \cdot \mathrm{CO}_{2} \mathrm{H}
\end{aligned}
$$

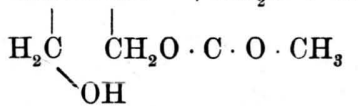

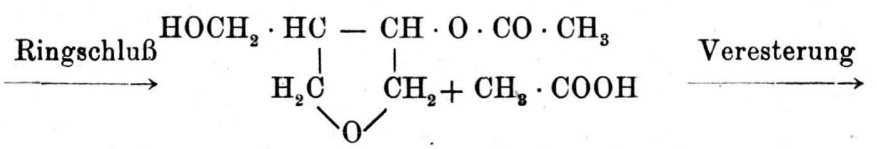

Selbstverständlich hätte man in den vorstehenden Formeln den Ringschluß schon unter B beim Buten-(2)-diol-(1.4)-monoacetat annehmen und die folgenden Umsetzungen dann am Grundkörper des 2.5-Dihydro-furans erklären können. Da wir dieses jedoch aus dem Reaktionsgemisch nicht isoliert haben und auch die Essigsäureanlagerung an das analoge Tetrahydrophthalan bisher nicht verwirklichen konnten, haben wir von dieser Deutungsmöglichkeit Abstand genommen. Die Formeln VI und VII sind aus der Synthese und der Analogie zu den Reaktionen des Tetrahydrobenzylacetates deduziert. Sie bedürfen daher noch der analytisch-präparativen Bestätigung. - Auch bei dieser Umsetzung haben wir kein Trimethylenglykol-diacetat erhalten.

10 Pr i n s, Chem. Weekbl. 14, 923 [1917]; 16, 1510 [1919]; Chem. Zbl. 1918 I, 168; 1920 I, 423; Koninkl. Akad. van Wetensch. Amsterdam, Wisk. en Natk. Afd. 27, 1496; Chem. Zbl. 1919 III, 1001; Recueil Trav. chim. Pays-Bas 51 ([4] 13), 469 [1932]; Chem. Zbl. 1932 II, 854.
IV. Abwandlung des Trimethylenglykol-diacetates

Nach unseren. am Hexahydrosaligenin-diacetat gewonnenen Erfahrungen ${ }^{2}$ lag es nahe, die thermische Essigsäureabspaltung auch auf das Trimethylenglykol-diacetat zur Darstellung von Allylacetat anzuwenden. Wir zersetzten Trimethylenglykol-diacetat im langsamen Stickstoffstrom bei $565^{\circ}$ mit dem erwarteten Erfolg. Im Hinblick auf die geringe Menge Ausgangsmaterial war die Ausbeute gut. Allylacetat konnte auch durch thermische Spaltung von Propylenglykol-diacetat erhalten werden.

Die Synthese des Allylacetates aus Äthylen gemäß der Reaktionsfolge I $\rightarrow$ II $\rightarrow$ IV darf als ein Kernstück angesehen werden zu jenen Verfahren, die die Darstellung des Glycerins über die Allylstufe verwirklichen, wodurch gleichzeitig ein neuer Weg zu dessen Totalsynthese offensteht. 
Bei einem Versuch, vom Trimethylenglykol-diacetat durch vorsichtige Chlorierung zum $\beta$-Chlor-diacetin $\mathrm{zu}$ gelangen, dessen Verseifung direkt zum Glycerin führen würde, erfolgte im Uviol-Licht rasche Chloraufnahme. Wohl infolge Úberdosierung entstanden im wesentlichen perchlorierte Produkte. Die Halogenierungsversuche werden fortgesetzt.

\section{Beschreibung der Versuche ${ }^{11}$}

Mitbearbeitet von $\mathrm{Hedwig} \mathrm{Padberg}$ und
Ursula Wei $\beta$

Äthylen-Paraformaldehyd in Eisessig

In einem 2-l-Autoklaven aus V2A-Stahl wird $\mathrm{zu}$ 200 g Paraformaldehyd, $800 \mathrm{~g}$ Eisessig und $28 \mathrm{ccm}$ konz. Schwefelsäure Äthylen bis zu einem Druck von 50 Atm. kalt aufgepreßt. Nun wird 3 Stdn. auf 130 bis $140^{\circ}$ erhitzt, wobei der Druck auf 73 Atm. ansteigt. Nach dem Erkalten beträgt der Druck 42 Atm. und ist nach weiterem 16-stdg. Erhitzen unverändert; man entspannt bis nahezu Atmosphärendruck und preßt erneut $48 \mathrm{Atm}$. frisches Äthylen nach. Nach 2-stdg. Erhitzen auf $130^{\circ}$ und Wiedererkalten ist der Druck auf $34 \mathrm{Atm}$. gesunken. Weiteres 8-stdg. Erhitzen bewirkt keinen Druckabfall. Entspannen, Nachpressen (45 Atm.) und Erhitzen werden noch einmal in gleicher Weise wiederholt. Nach 5-stdg. Erhitzen beträgt der Druck im erkalteten Autoklaven 35 Atm. und bleibt hier bei weiterem 23-stdg. Erhitzen praktisch konstant. Das Reaktionsgemisch, eine braun-schwarze, lauchartig riechende Flüssigkeit, wird über Glaswolle filtriert, mit $106 \mathrm{~g}$ wasserfreier Soda versetzt und durch Destillation bei $744 \mathrm{~mm}$ in folgende Fraktionen zerlegt:

1. 57 bis $100^{\circ}$, farbl. Flüss.,

2. 100 bis $114^{\circ}$, farbl. Flüss.,

3. Rückstand braunes öl.

Frakt. 1 wird im Scheidetrichter mit gesättigter Sodalösung geschüttelt. Nach dem Absetzen hat sich an der Flüssigkeitsoberfläche eine farblose Schicht abgeschieden. Diese wird mit gesättigter Natriumsulfatlösung gewaschen und über wasserfreiem Natriumsulfat getrocknet. Die Destillation ergibt ca. $17 \mathrm{~g}$ einer farblosen Flüssigkeit vom Sdp.743 55 bis $75^{\circ}$.

Daraus werden durch wiederholte Destillation 3 Fraktionen gewonnen:

1a. Sdp. . $_{52} 5$ 5, 3 bis $56^{\circ}$, farbl. Fl., V.Z. 765,4, S.Z. 0, 2a. $\quad 56.58^{\circ}, \quad \mathrm{C} 47,59 \%, \mathrm{H} 8,31 \%$ 3a. C $47,52 \%, \mathrm{H} 8,29 \%$

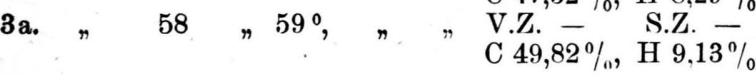

Berechnet für Methylacetat $\mathrm{C}_{3} \mathrm{H}_{6} \mathrm{O}_{2}$ : C 48,65\%, $\mathrm{H} 8,11 \%$ V.Z. 758,2

Fra k t. 2 ist nicht aufgearbeitet worden.

Der R ü c k st a n d 3 wird in Äther aufgenommen. Die Atherlösung wird filtriert und so lange mit gesättigter

11 Alle angegebenen Schmelz- und Siedepunkte sind unkorrigiert.
Sodalösung geschüttelt, bis die wäßrige Schicht schwach alkalisch reagiert, mit gesättigter Natriumsulfatlösung neutral gewaschen und über Natriumsulfat getrocknet. Nach dem Abdestillieren des Äthers erhält man durch wiederholte Destillation an der Wi d me r - Spirale folgende Eraktionen:

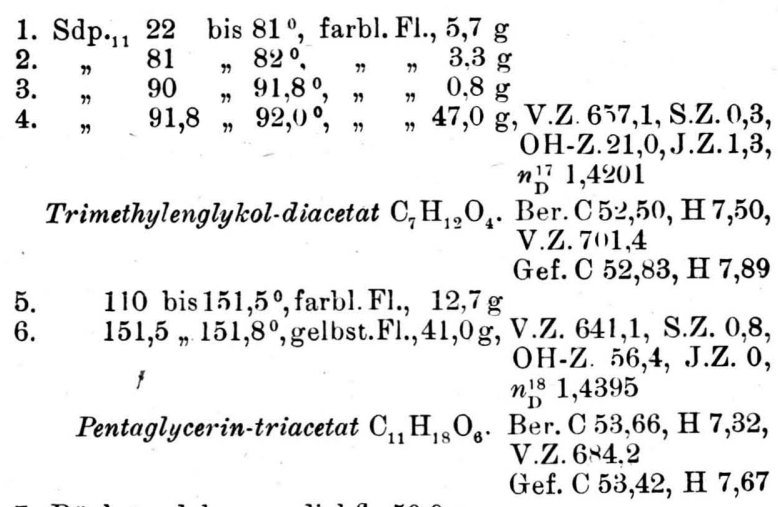

7. Rückstand braun, dickfl. 50,0 g

Trimethylenglykol (IV) aus dem Diacetat

1. Mit methylalkohol. Kalilauge: 9,3 g Trimethylenglykol-diacetat (nach der vorstehend beschriebenen Methode hergestellt: V.Z. 670, S.Z. 0,2, J.Z. 0,2. C 52,98\%, H 7,77\%) werden mit überschüssiger methylalkohol. Kalilauge 2 Stdn. unter Rückfluß gekocht. Der Methylalkohol wird abdestilliert und der Rückstand mit verd. Schwefelsäure neutralisiert. Man dampft auf dem Wasserbade bis fast zur Trockne ein und extrahiert das zurückbleibende Salzgemisch mehrfach mit Alkohol. Nach Vertreiben des Alkohols erhält man durch Destillation bei $10 \mathrm{~mm}$ eine etwas gelbstichige Flüssigkeit, die im wesentlichen bei etwa $105^{\circ}$ übergeht. Sie schmeckt intensiv süß. $n_{\mathrm{D}}^{20} 1,4355$.

Das Bis-phenylurethan schmilzt nach 4-maligem Umkristallisieren aus Benzol zwischen 136,5 bis $137,5^{\circ}$.

$\begin{array}{lllll}\mathrm{C}_{17} \mathrm{H}_{18} \mathrm{O}_{4} \mathrm{~N}_{2} . & \text { Ber. } & \text { C 64,97, } & \mathrm{H} 5,73, & \text { N } 8,92 . \\ & \text { Gef. } & \text { C 65,51, } & \text { H 6,09, } & \text { N 8,86. }\end{array}$

2. Mit methylalkohol. Salzsäure: $13,7 \mathrm{~g}$ Trimethylenglykol-diacetat (Sdp.11 91,8 bis $92^{\circ}$, V.Z. 657,1, S.Z. 0,3, OH-Z. 21,0, J.Z. 1,3) werden mit $20 \mathrm{ccm} 3$-proz. methylalkohol. Salzsäure in einem $\mathrm{Cl}$ a is e n-Kolben mit schräg nach oben gestelltem Kühler 1 Stde. gekocht. Dann destilliert man $18 \mathrm{ccm}$ Lösungmittel ab, fügt zwecks vollständiger Umsetzung $15 \mathrm{ccm}$ 1-proz. methylalkohol. Salzsäure wieder hinzu und erhitzt erneut $1 / 2$ Stde. in der beschriebenen Weise. Nach Vertreiben des Lösungsmittels wird bei $12 \mathrm{~mm}$ destilliert. Nach einem geringen Vorlauf $(0,9 \mathrm{~g})$ geht das Glykol als farbloses, dickflüssiges Öl etwas trübe bei 110 bis $111 \% / 12 \mathrm{~mm}$ über. Ausb. 5,1 g. Es wird noch einmal destilliert: Sdp.11 108 bis $108,2^{\circ} \cdot n_{\mathrm{D}}^{11,5} 1,4389$.

Bis-phenylurethan aus Methanol, Schmp. 136 bis $138,2^{\circ}$. Mischschmelzpunkt mit einem aus Trimethylenbromid $(\mathrm{K}$ a h l b a u m) bereiteten Präparat zeigt keine Depression. 
Trimethylenglykol-diacetat a us Trimethylenbromid ${ }^{12}$

$144 \mathrm{~g}$ frisch geschmolzenes, feinpulverisiertes Kaliumacetat werden mit $202 \mathrm{~g}$ Trimethylenbromid (K ahlbaum) und $64 \mathrm{~g}$ Eisessig vermischt und $\mathbf{3}^{1} / \mathbf{2}$ Stdn. unter Rückflußkühlung und gelegentlichem Umschwenken gekocht. Die erste Destillation ergibt 162 g Destillat vom Sdp.759 120 bis $208^{\circ}$. Nach 3-maliger Fraktionierung an der W id m e r-Spirale gewinnt man $72 \mathrm{~g}$ Diacetat als farblose Flüssigkeit vom Sdp.11 $92^{\circ} \cdot n_{\mathrm{D}}^{\mathbf{1 7}} \quad 1,4192$.

Das hieraus mit methylalkohol. Salzsäure hergestellte Trimethylenglykol erwies sich in allen Eigenschaften als identisch mit dem aus dem Autoklavenversuch stammenden.

Pentaglycerin (V) a us dem Triacetat

1. Mit methylakohol. Kalilauge: $14,7 \mathrm{~g}$ Pentaglycerintriacetat (V.Z. 646,0, S.Z. 0,3, J.Z. 0,6; gef. C 53,33\%, H 7,36\%) werden mit überschüssiger methylalkohol. Kalilauge durch 2-stdg. Kochen verseift. Nach dem Abdestillieren des Methylalkohols wird mit verd. Schwefelsäure neutralisiert und auf dem Wasserbade bis fast zur Trockne eingedampft. Der Eindampfrückstand wird im Soxhlet mit Äther (worin die Substanz sehr schwer löslich ist) während 10 Stdn. möglichst erschöpfend extrahiert. Man erhält 4,5 g einer kristallinen Substanz vom Schmp. 190 bis $194^{\circ}$. Nach mehrmaligem Umkristallisieren aus Alkohol-Äther ist das Präparat vollkommen farblos und schmilzt zwischen 199 bis $200^{\circ}$. Es schmeckt süß und ist sublimierbar.

$\mathrm{C}_{5} \mathrm{H}_{12} \mathrm{O}_{3}$. Ber. C 50,00, $\mathrm{H} \mathrm{10,00.}$

Gef. C 50,10 , H 9,85 .

2. Mit methylalkohol. Salzsäure: $10 \mathrm{~g}$ Pentaglycerintriacetat (V.Z. 641,1, S.Z. 0,8, OH-Z. 56,4, J.Z. 0; gef. C $53,42 \%, \mathrm{H} 7,67 \%$ ) werden mit 30 ccm 1-proz. methylalkohol. Salzsäure 2 Stdn. gekocht. Nach dem Vertreiben des Lösungsmittels hinterbleibt ein gelbes öl, das beim Erkalten erstarrt. Man nimmt in wenig absol. Methanol auf und fällt mit wasserfreiem Äther. Die Ausbeute ist nahezu quantitativ. Aus Methanol-Äther umkristallisiert: Schmp. 199 bis 200,5 .

Essigsäureabspaltung aus Trimethylenglykol-diacetat durch konz.

S c h w e f e ls äu re

$20 \mathrm{~g}$ Trimethylenglykol-diacetat (Sdp.11 91,8 bis $92^{\circ}$, V.Z. 657,1, S.Z. 0,3, OH-Z. 21,0, J.Z. 1,3) werden mit $1 \mathrm{ccm}$ konz. Schwefelsäure an einer kleinen Kolonne bei $745 \mathrm{~mm}$ destilliert. Das Destillat geht im wesentlichen zwischen 95 und $105^{\circ}$ über. Sobald die Destillation träge wird oder die Destillationstemperatur wesentlich steigt, gibt man jeweils 0,5 bis $1 \mathrm{ccm}$ konz. Schwefelsäure zu. Das Destillat (17,8 g) wird in $25 \mathrm{ccm}$ Äther aufgenommen und, wie beschrieben, mit gesättigter Soda- und Natriumsulfatlösung behandelt

12 Vorschrift von I p a t o w, vergl. Chem. Zbl. 1914 I, 2161. und über Natriumsulfat getrocknet. Nach Abdestillieren des Äthers gehen zwischen 70 und $100^{\circ} \mathrm{ca} .3 \mathrm{ccm}$ einer farblosen Flüssigkeit von kräftigem Geruch nach Allylacetat über. Ausb. nach nochmaliger Destillation: ca. $1 \mathrm{ccm}$, Sdp.740 $100^{\circ}, n_{\mathrm{D}}^{18,2} 1,3998$.

\section{Umsetzung von Allylacetat mit Eisessig}

1. In 2 Einschmelzrohren werden je $20 \mathrm{~g}$ Allylacetat mit 40 g Eisessig und $1 \mathrm{ccm}$ konz. Schwefelsäure 6 Stdn. auf 160 bis $200^{\circ}$ erhitzt. Beim Öffnen der Rohre macht sich kein Überdruck bemerkbar. Die dunklen Flüssigkeiten beider Rohre werden vereinigt und filtriert. Das Filtrat wird nach Zugabe von $6 \mathrm{~g}$ wasserfreier Soda durch Destillation weitgehend von nicht umgesetztem Allylacetat und überschüss. Essigsäure befreit. Den Rückstand nimmt man in Äther auf und behandelt die Ätherlösung wie üblich. Nach Abdestillieren des Äthers ergibt die Vakuumdestillation $11,5 \mathrm{~g}$ eines farblosen Öles vom Sdp.10 75 bis $80^{\circ}$ (Propylenglykol-diacetat). Dieses wird mit methylalkohol. Salzsäure verseift. Nach 2-maliger Fraktionierung erhält man 4,5 g eines fast farblosen, dickflüssigen Öls vom Sdp.9 82 bis $82,5^{\circ} . n_{\mathrm{D}}^{11,5} 1,4323$.

\section{Propylenglykol $\mathrm{C}_{3} \mathrm{H}_{8} \mathrm{O}_{2}$. Ber. $\mathrm{C}$ 47,37, $\mathrm{H} 10,53$. Gef. C 47,26, H 10,58.}

2. $20 \mathrm{~g}$ Allylacetat werden mit $40 \mathrm{~g}$ Eisessig unter Zugabe von 2 g gepulvertem Ferrosulfat und 3 Tropfen konz. Schwefelsäure im Rohr 6 Stdn. auf 150 bis $170^{\circ}$ erhitzt. Die Aufarbeitung erfolgt wie voranstehend. Man erhält ca. 5 ccm eines dunkelbraunen Öls, das bei der Destillation eine farblose Flüssigkeit vom Sdp. 84 bis $85^{\circ} / 13 \mathrm{~mm}$ liefert. Es hinterbleibt praktisch kein Rückstand.

\section{Allylalkohol-Paraformaldehyd in Eisessig}

Ein Gemisch aus $70 \mathrm{~g}$ Paraformaldehyd, $500 \mathrm{~g}$ Eisessig und $10 \mathrm{ccm}$ konz. Schwefelsäure wird unter lebhaftem Rühren bis zur klaren Lösung erhitzt. Man fügt dann bei ca. $60^{\circ} 116 \mathrm{~g}$ Allylalkohol reinst (K a h l b a m m) hinzu und erhitzt unter Rückflußkühlung vorsichtig zum Sieden. Nach 6-stdg. Kochen wird das braun-schwarze Reaktionsgemisch in $200 \mathrm{ccm}$ Äther aufgenommen und die Ätherlösung wie beschrieben behandelt. Nach dem Abdestillieren des Äthers liefert die Rohdestillation folgende Fraktionen:

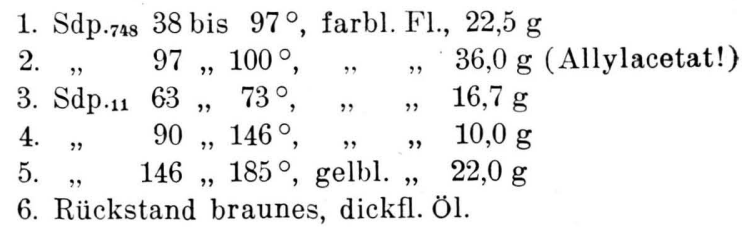

Aus den Frakt. 3 bis 5 erhält man durch sorgfältige Fraktionierung an der Widmer-Spirale im wesentlichen nur zwei Fraktionen: 
1 a) Sdp.10 63,2 bis $63,5^{\circ}$, farbl. Fl., V.Z. 418,9 (Theorie 431,6),

S.Z. 1,0, OH-Z. 11,4, J.Z. 2,2.

$$
\begin{aligned}
& \text { 3-Acetoxy-tetrahydrofuran (?) } \\
& \mathrm{C}_{6} \mathrm{H}_{10} \mathrm{O}_{3} \text {. Ber. C 55,37, H 7,75. } \\
& \text { Gef. C 55,10, 55,24, H 7,89, 7,72. }
\end{aligned}
$$

2 a) $\mathrm{Sdp}_{\cdot 10,5} 146$ bis $146,5^{\circ}$, farbl. Fl.,

V.Z. 551,3 (Theorie 554,9)

S.Z. 0,2 , OH-Z. 16,0, J.Z. 10,1

\section{3-Acetoxy-4-acetoxymethyl-tetrahydrofuran}

$$
\begin{array}{llll}
\mathrm{C}_{\mathbf{9}} \mathrm{H}_{\mathbf{1 4}} \mathrm{O}_{5} . & \text { Ber. } & \text { C 53,46, } & \text { H } 6,98 . \\
& \text { Gef. } & \text { C 53,48, 53,49, } & \text { H 7,15, 7,18. }
\end{array}
$$

Thermische Essigsäureabspaltung a us Trimethylenglykol-diacetat

Apparatur: Ein $100 \mathrm{ccm}-$ Rundkolben aus Quarz (A) mit seitlichem Ansatzrohr zum Einleiten von Stickstoff ist durch Schliff mit einer senkrecht darüber stehenden, elektrisch heizbaren Zersetzungskolonne aus Duranglas (Durchm. 1,7 cm, Länge insgesamt - $60 \mathrm{~cm}$, Heizlänge $40 \mathrm{~cm}$ ) verbunden. Die Kolonne ist mit kleinen Quarzstückchen gefüllt. In der Mitte des beheizten Rohrzylinders befindet sich, im Füllmaterial eingebettet, die Kugel eines Stab-Quarzthermometers, das außerhalb der Säule abgelesen werden kann. An einen kurzen Destillierstutzen am oberen Teil der Kolonne ist ein Liebig-Kühler angeschlossen, der in einen Vakuumvorstoß einmündet. Der Vorstoß reicht in eine Vorlage (B), die mit Eiswasser gekühlt wird. Der Vakuumstutzen des Vorstoßes steht in Verbindung mit einer Waschflasche (C), die eine gesättigte Natriumbisulfit-Lösung enthält und einerseits zur Absorption von evtl. entstehendem und in der Vorlage A nicht kondensiertem Acrolein, andererseits zur Verhütung des Eindringens von Luft in die Apparatur vorgeschaltet ist. Sämtliche Verbindungen sind luftdicht eingepaßt.

Versuch: In A befinden sich $16 \mathrm{~g}$ Trimethylenglykoldiacetat (aus Trimethylenbromid, Sdp.11 $92^{\circ}$ ). Der seitliche Ansatz des Kolbens wird mit einem einer Stickstofflombe angeschlossenen Blasenzähler verbunden und durch die gesamte Apparatur bei geheizter Kolonne $\left(565^{\circ}\right) 1 / 2$ Stde. lang ein lebhafter Stickstoffstrom geleitet. Nachdem so der in der Apparatur befindliche Luftsauerstoff völlig entfernt ist, drosselt man den Stickstoffstrom ein wenig und beginnt, $\mathrm{A}$ in einem Metallbad allmählich auf Siedetemperatur zu erhitzen. Durch Regulierung der Temperatur des Metallbades und der Stickstoffzufuhr lenkt man den Versuch so, daß die Zersetzung kontinuierlich und möglichst vollständig vonstatten geht. Der Eintritt der Zersetzung macht sich dadurch bemerkbar, daß sich am Destillierstutzen der Kolonne Nebel bilden, die bei richtiger Durchführung des Versuches im Kühler vollständig kondensiert werden. Die mittlere Badtemperatur beträgt etwa $215^{\circ}$; gegen Ende des Versuches wird sie bis auf $300^{\circ}$ erhöht. Die Temperatur der Kolonne läßt sich mit Hilfe eines Regulierwiderstandes ohne Schwierigkeiten bei $565^{\circ}$ konstant halten. Nach 4-stdg. Versuchsdauer ist der Quarzkolben leer. In der Vorlage B befinden sich $13,25 \mathrm{~g}$ einer gelben Flüssigkeit. Das Gewicht der Waschflasche mit Absorptionsflüssigkeit ist praktisch unverändert.

Das Destillat wird mit $10 \mathrm{ccm}$ Äther versetzt und die Ätherlösung in bekannter Weise zur Destillation vorbereitet. Die erste Destillation ergibt ca. $5 \mathrm{~g}$ einer farblosen Flüssigkeit, die bei $748 \mathrm{~mm}$ im wesentlichen zwischen 95 und $100^{\circ}$ übergeht. Sie riecht nach Allylacetat. Bei nochmaliger Destillation erhält man ein Präparat vom Sdp.748 95 bis $102^{\circ}, n_{\mathrm{D}}^{1 \mathrm{~s}}$ 1,4046. Zum Vergleich wird ein aus reinstem Allylalkohol ( $\mathrm{K}$ a h lb a um), Eisessig und Schwefelsäure hergestelltes Präparat (Sdp.749 101 bis $103^{\circ}$ ) herangezogen: $n_{1}^{18}$ 1,4039 .

Nach Mitteilung von Hrn. Prof. F e hér stimmen beide Präparate in den wesentlichen $\mathrm{R}$ a ma $\mathrm{n}$ - Linien überein.

\section{Propylenglykol-diacetat}

30 g i-Propylenglykol (Sdp.11 86 bis $88^{\circ}$ ) werden mit 150 ccm Essigsäureanhydrid 6 Stdn. unter Rückfluß gekocht. Nach 2-malizer Fraktionierung liegt ein Präparat vom Sdp. 76 bis $79^{\circ}$ vor, das zur Beseitigung von Anhydrid mit Soda- und Natriumsulfatlösung behandelt und erneut destilliert wird. Das Diacetat geht dann bei $80 \% / 11 \mathrm{~mm}$ über. $n_{\mathrm{D}}^{17} 1.4148$.

Thermische Essigsäureabspaltung aus Propylenglykoldiacetat

20 g Propylenglykol-diacetat werden in der gleichen Weise wie das Trimethylenglykol-diacetat bei $575^{\circ}$ innerhalb von $2^{1 / 2}$ Stdn. zersetzt. Man erhält $17,4 \mathrm{~g}$ eines gelben, stechend riechenden Destillates, das fuchsinschweflige Säure rötet. Man schüttelt wiederholt mit Natriumbisulfitlösung durch, wobei sich die untere Schicht in abnehmender Stärke orange färbt. Die organische Schicht wird in $10 \mathrm{ccm}$ Äther aufgenommen und die Ätherlösung wie üblich gewaschen und getrocknet. Nach Abdestillieren des Äthers erhält man 2,2 g einer farblosen, nach Allylacetat riechenden Flüssigkeit vom Sdp.739 80 bis $100^{\circ} . n_{1}^{18} 1,4069$. Das Präparat ist noch nicht einheitlich.

\section{Chlorierung von Trimethylenglykol-} di a c e tat

In einem 150-ecm-Quarzkolben mit seitlichem Ansatz und aufgesetztem Rückflußkühler mit Chlorcalciumrohr befinden sich $20 \mathrm{~g}$ Trimethylenglykol-diacetat (aus dem Trimethylenbromid, Sdp.11 $92^{\circ}$ ). Durch den seitlichen Ansatz des Quarzkolbens leitet man mit Hilfe eines dünnen Rohres bei Zimmertemperatur einen lebhaften Strom trockenen Chlors ein. Die Flüssigkeit nimmt bald die Farbe des elementaren Chlors an, und auch nach ${ }^{1} / \mathrm{g}_{\mathrm{g}}$-stdg. Einleiten ist keine Entfärbung zu beobachten. Bestrahlt man nun aus etwa $30 \mathrm{~cm}$ Abstand 
mit einer starken Quecksilberquarzlampe, so tritt sofort Entfärbung und Chlorwasserstoffentwicklung ein. Während des Einleitens bleibt die Flüssigkeit unter Selbsterwärmung völlig farblos. Nach 60 Min. beträgt die Gewichtszunahme des Kolbens 5 g. Bei einer Gewichtszunahme von insgesamt $10 \mathrm{~g}$ wird die Chlorierung abgebrochen. Man erhält ein farbloses öl, das in Wasser sofort zu Boden sinkt. Das Reaktionsgemisch läßt sich an einer kleinen Kolonne nicht unzersetzt destillieren. Während der Destillation beobachtet man mehr oder minder starke Nebelbildung:
1. Sdp. 75547 bis $95^{\circ}$, farbl. Fl., $7 \mathrm{~g}$

2. „ 95 bis $105^{\circ}$, gelbl. Fl., $10,1 \mathrm{~g}$ (Zersetzung!)

3. „ 80 (Zers.!) bis $138^{\circ}$, gelbl. Fl., $1,8 \mathrm{~g}$

4. Rückstand schwarz, verkohlt.

Frl. Gertrud M e r k el danken wir für die Ausführung der chemischen Kennzahlanalysen. Hr. Prof. $\mathrm{F}$ e h é $\mathrm{r}$ und Frl. $\mathrm{B}$ a u d l e $\mathrm{r}$ hatten die Liebenswürdigkeit, das aus der thermischen Zersetzung des Trimethylenglykol-diacetates erhaltene Allylacetat einer $\mathrm{R}$ a m a n-Analyse zu unterziehen. Auch ihnen sei an - dieser Stelle bestens gedankt.

\title{
Über ein einfaches Verfahren zur Darstellung freier Aminosäureester aus den Esterhydrochloriden
}

\author{
Von Günther Hillmann \\ Aus dem Kaiser-Wilhelm-Institut für Biochemie, Berlin-Dahlem, jetzt Tübingen
}

(Z. Naturforschg. 1, 682-683 [1946]; eingegangen am 25. Juli 1946)

$\mathrm{F}$ ür die Synthese von Peptiden sind meist freie Aminosäureester erforderlich, für deren Darstellung aus den Esterhydrochloriden eine Reihe von Methoden zur Verfügung stehen, die aber z. Tl. umständlich sind oder unbefriedigende Ausbeute liefern. Bei dem bekannten Verfahren von E. Fischer ${ }^{1}$, das auf der Freisetzung von Aminosäureestern durch $\mathrm{NaOH}$ und $\mathrm{K}_{2} \mathrm{CO}_{3}$ beruht, ist die Ausbeute gering (durchschnittlich ca. 50\%). Th. Curtius ${ }^{2}$ stellte die freien Aminosäureester durch Behandlung der Hydrochloride mit $\mathrm{Ag}_{2} \mathrm{O}$ bzw. $\mathrm{AgOH}$ her. Trotz der Modifizierung durch S. Ai und S. Ushida ${ }^{3}$ scheint das Verfahren wegen der Verwendung von $\mathrm{Ag}_{2} \mathrm{O}$ wenig geeignet. Nach E. Abderhalden ${ }^{4}$ werden die Esterhydrochloride mit der genau berechneten Menge Natriumalkoholat umgesetzt; dazu sind relativ große Mengen von absol. Alkohol und Äther notwendig. Beim Abdestillieren des Alkohols werden die Ester der niedrigmolekularen Aminosäuren teilweise mitgerissen. Bei der Einwirkung von $\mathrm{BaO}$ bzw. $\mathrm{Ba}(\mathrm{OH})_{2}$ auf die Esterhydrochloride in Chloroform nach P. A. Levene ${ }^{5}$ wird ein Teil des entstandenen Esters wieder verseift; das gilt vor allem für die besonders alkaliempfindlichen Aminodicarbonsäureester. Nach J. A. B ach$\mathrm{mann}^{6}$ wird diese Fehlerquelle zwar weitgehend durch die Umsetzung mit $\mathrm{BaO}$ in absol. Äther ausgeschaltet, das Verfahren ist jedoch sehr zeitraubend.

Es wurde nun gefunden, daß diese Schwierigkeiten bei der Herstellung freier Aminosäureester sich durch die Umsetzung der Esterhydrochloride mit ammoniakalischem Chloroform vollständig ausschalten lassen. Während die Aminosäureester mit ammoniakalischem

1 Ber. dtsch. chem. Ges. 34, 436 [1901].

2 J. prakt. Chem. [2] 37, 159 [1888].

3 J. Soc. chem. Ind. Japan [Suppl.] 36, 221 [1933].

4 Hoppe-Seylers Z. physiol. Chem. 120, 208 [1922].
Alkohol unter Amidbildung ${ }^{7}$ reagieren, tritt diese Reaktion mit ammoniakalischem Chloroform in der Kälte nicht ein.

Alle Aminosäureester-hydrochloride weisen eine gewisse Löslichkeit in Chloroform auf. Die Umsetzung mit Ammoniak zu freien Aminosäureestern und Ammoniumchlorid findet infolgedessen rasch statt. Da hierbei keine Bildung von Wasser eintritt, besteht keine Gefahr einer Verseifung der freien Ester.

Die Anwendung genau berechneter Mengen von Ammoniak ist nicht erforderlich, da auch bei Vorhandensein eines geringen Utberschusses an Ammoniak unter den ausgearbeiteten Versuchsbedingungen in Chloroform keine Amidbildung stattfindet.

Das bei der Umsetzung entstehende Ammoniumchlorid ist in Chloroform unlöslich und läßt sich bequem abfiltrieren bzw. absaugen. Hierdurch erübrigt sich ein Zusatz von Äther zur besseren Abscheidung, wie es bei der Abderhaldenschen Methode für die Ausfällung des gebildeten Natriumchlorids notwendig ist. Beim Abdestillieren des Chloroforms gehen nur sehr geringe Mengen der niedrig siedenden Aminosäureester mit über. Die Ausbeute ist praktisch quantitativ. Sofern die jeweiligen Versuchsbedingungen bei der Synthese von Peptiden die Verwendung von Lösungen der Aminosäureester in Chloroform zulassen, sind nach dem Abfiltrieren des gebildeten Ammoniumchlorids und der Entfernung des üherschüssigen Ammoniaks im Vakuum die Chloroformlösungen der freien Aminosäureester nach unseren Erfahrungen direkt zur Synthese geeignet.

5 J. Biol. Chem. 81, 699; 86, 419 [1929].

6 Dissertat. Eidgen. T. H. Zürich, Agriculturchem. Labor. 1927.

7 Schenk, Arch. Pharmaz. Ber. dtsch. pharmaz. Ges. 247, 510 [1909]. 